\title{
OAP: Toward a Process for an Open World
}

\author{
Yuanzhi Wang \\ Department of Computer Science \\ The Australian National University, Australia \\ derek. wang@anu. edu . au
}

\begin{abstract}
To deal with increasing complexity, volatility, and uncertainty, a process for engineering software-intensive systems in an open world, called Organic Aggregation Process (OAP), is proposed. It is based on a concept called "Organic Aggregation", and consists of a coherent and flexible aggregation of carefully-categorised, hierarchically organised, and inter-connected activities. It supports flexible process aggregations via a capacity reuse mechanism called Epitome. A modeldriven method and a supporting tool environment are integrated into OAP, which enables efficient manipulation and utilisation of OAP models, and automatic generation of concrete systems. It supports, and promotes a focus on, high-level intellectual efforts such as perception of reality, rationalisation of problem situations, and derivation of "soft" desired ends. A proof-of-concept case study is briefly presented in this paper to illustrate the application of OAP approach in a real world setting.
\end{abstract}

Keywords: Process, Software Engineering, Software Process, MDE.

\section{Introduction}

The variety, complexity, and dynamic of software-intensive systems have been significantly increased because of advances in technologies and society in the open environments of present era. Owing to increasing complexities, volatility, and uncertainty involved in an open world, the well-known gap between computing capabilities and usage demands has reached a critical level that hinders successful engineering of complex systems in such an environment [1. Therefore, it becomes pressingly necessary to assess the problems of current engineering approaches and to explore different ways to address such a great challenge.

In this paper, an engineering process called Organic Aggregation Process, or OAP for short, is proposed. It consists of a set of inter-connected activities that are organised in a coherent and flexible hierarchy. This structure aims to form a coherent linkage between human intellectual efforts and practical reality. That is, a path from human perception of complex problem situations to sensible desired engineering ends, and to practical engineering actions that eventually improve the situations. Such a linkage can be aggregated organically through a capacity reuse mechanism, in support of organic agility. Moreover, a model-driven method and some supporting tools are integrated into OAP, and facilitate management of its engineering activities in a dynamically complex environment.

J. Münch, Y. Yang, and W. Schäfer (Eds.): ICSP 2010, LNCS 6195, pp. 175-187, 2010.
(c) Springer-Verlag Berlin Heidelberg 2010 
Briefly, by synthesising a coherent and flexible conceptual structure, a modeldriven methodology, and an integrated tool environment, OAP aims to: 1) promote a focus on higher-level intellectual efforts within a cognitive world of mind, especially perception of reality, rationalisation of problem situations, and derivation of "soft" desired ends; and 2) co-ordinate conduction of various activities in a coherent, flexible, and reusable means towards systematic generation of software systems that improve the perceived problematic situations.

The remainder of the paper is organised as follows: section 2 briefly analyses existing process models of engineering and problem-solving; section 3 presents the OAP process in terms of its coherent and flexible conceptual structure, modelling mechanism, and integrated tool environment; section 4, using a proof-ofconcept case study, illustrates how OAP approach is applied in a real-world setting to meet its objectives ; section 5 analyses the positive results, contribution, and limitation of this work based on the case study; Finally, section 6 presents a summary of this paper and concludes some contributions of this work.

\section{Background and Related Work}

The origin of a notion of processes can be traced back to ancient Greek philosopher Heraclitus, who held that the essence of the world is not material substance of things but rather a continuous natural process, or "flux" in his term 2. Whitehead's definition of a process is "a sequentially structured sequence of successive stages or phases" 3. In the context of software engineering, Fuggetta defined a process as "the coherent set of policies, organisational structures, technologies, procedures, and artefacts that are needed to conceive, develop, deploy, and maintain a software product" 4]. These definitions all involve a concept of life-cycle, and some composing elements with a structure of certain shape or format.

Similarly, Royce's influential waterfall model 5 is a software process that consists of a sequence of activities such as requirements analysis, design, and implementation. This approach gradually evolved into more iterative, incremental, and evolutionary models, sometimes called Iterative and Incremental Development (IID) [6], such as spiral model, rapid prototyping, Rational Unified Process (RUP), and more recently, agile methodologies. John Boyd's Observation, Orientation, Decision, and Action (OODA) loop, as a decision-making process model, was designed to cope with highly dynamic and competitive environments by accelerating a person's own loop and weakening opponents' abilities to complete their loops at faster paces [7. This model has been successfully adopted in critical and dynamic environments such as military battles and business competition.

This paper, however, argues that these approaches are inadequate to deal with great challenges of complexity, volatility, and uncertainty, which are commonly involved in open environments. For example, OODA model and its followers, in general, are too abstract and coarse-grained, and lack some fundamental elements in an engineering context, such as clearly defined activities at detail level, their boundaries and relationships, and supporting tools. It provides neither guidelines with regards to internal structures and content, nor representational 
formalisms for each phase, in a concrete sense. Application of such a process is hence not controllable in an engineering context.

More importantly, after decades of evolution, these process models are still fundamentally based on the same concept, that is, pre-defined decomposition and refinement of life-cycle at fixed levels and rigid activity dependencies, which is referred as rigid plan-driven processes in this paper. For example, most IID processes have the same pre-defined phases such as Analysis, Design, Implementation, Test, and Verification, although at different paces, or sometimes with feedback added to different phases in the loop 6. Similarly, RUP comprises Inception, Elaboration, Construction and Transition. Whereas agile methodologies normally consist of Exploration, Planning, Iteration and Release 8]. So does OODA model and its followers.

These rigid plan-driven processes, although effective in some complex and safety-critical systems, lack flexibility and agility because they are often timeconsuming to carry out in the first place, drawn in time or quickly become out of synchronisation, and are difficult to change over time 9. Although agile methodologies aim to address this issue by using a lightweight process with shorter iterative development cycles and quick feedback, they lack order and cohesion in complex situations, and therefore are usually used only for small and medium sized projects [9].

\section{OAP Process Model for Software-Intensive Systems Engineering}

OAP approach is fundamentally based a concept called Organic Aggregation with an aim of addressing the grand challenges of an open world. An aggregation means a synthetic approach to look at the world that is aggregated by interrelated elements in a hierarchy [10]. Being organic means being coherent, ordered, sensible, and more importantly, being flexible enough to be able to continuously evolve towards a new favourable order from emerging chaos, at a pace faster than the changing rate of chaotic situation. Organic Aggregation therefore means creative synthesis of systems, such as objects, events, and processes, that is in a coherent order, and capable of coping with ever-changing chaotic environments.

Specifically, this concept is applied to not only the structure and interrelationships of process, but also its composing sub-processes, subordinate activities, and the products they produce. That is, a process is an organic aggregations of inter-related internal elements that are arranged in a coherent, efficient, and flexible way. In the meantime, capabilities to carry on sub-processes or internal activities, as well as the products they produce, such as various models and artefacts, also form organic aggregations that can be used, or reused, to aggregate other systems. These concepts are incorporated into the coherent structure of $\mathrm{OAP}$ and its mechanism to reuse knowledge and capacities, and are illustrated in the following sections. 


\subsection{The Coherent Structure of OAP}

OAP is coherently organised as a coherent hierarchy that has multiple levels. Its structure at a fundamental level is depicted on the left-hand side of figure 1. The central thick line represents engineering experience that provides an interaction interface between the world of Reality and Mind. It helps to distinguish human intellectual efforts from externally observable reality. On the one hand, the world of Mind involves important intellectual and cognitive activities that are essential precondition of problem-solving in complex problematic or chaotic engineering situation. On the other hand, since activities within the world of Reality are closer to physical environments, and are better understood and controllable compared with mental activities, they can be reproduced, automated, or controlled in a relatively easy way.
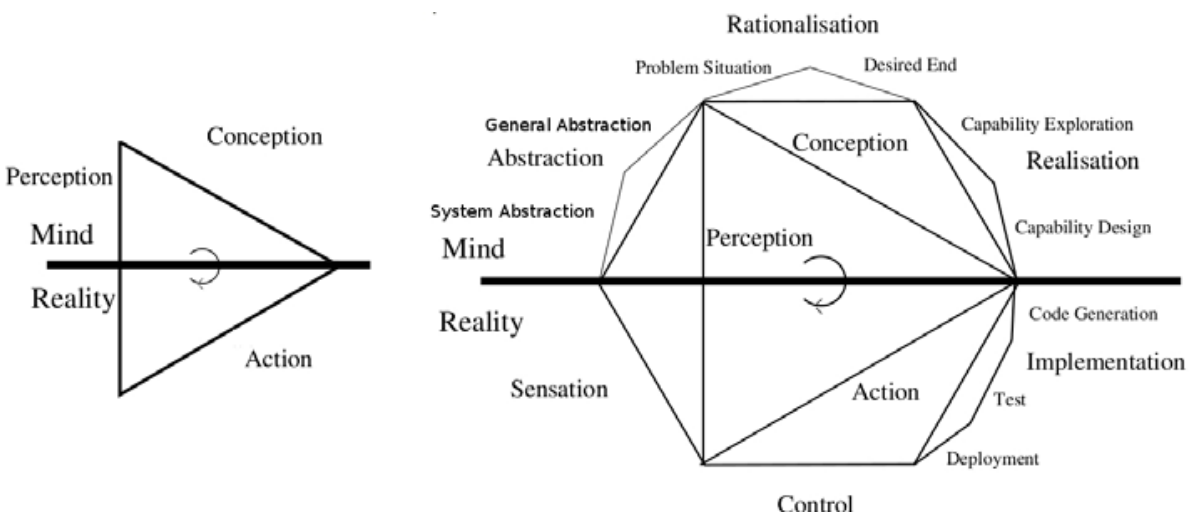

Fig. 1. A coherent conceptual structure of Organic Aggregation Process (OAP)

At this level, OAP consists of three interconnected activities, namely Perception, Conception, and Action, represented by straight lines with name beside them. These activities, collectively, connect the world of Mind with Reality. Perception is a set of activities that acquire information or knowledge about the Reality, and derive relevant representations in the world of Mind, which can be located, used, and reused by successive activities. Conception is human intellectual activities in the scope of Mind, which involve reasoning, rationalisation, decision-making, or plan-making sub-processes. Action, conducted in the world of Reality, deals with problematic situations by doing things, reacting to events, and other interactions with the outside worlds, according to outputs from Perception and Conception, such as knowledge of involved situations, desired ends, rules and laws, designs and plans. These activities are interconnected with each other and form continuous and iterative forward loops.

Moreover, activities at this level are composed of subordinate activities at more detailed levels, as depicted on the right-hand side of figure 1. Perception consists of Sensation and Abstraction. The former involves activities that directly 
collect required data from Reality, such as physical measurement of temperature and humidity, or real-time network performance monitoring. Whereas Abstraction, occurring in the world of Mind, is a type of activities that generalise or interpret the data or information collected by Sensation, and produce representations of relevant Reality in Mind. A collection, or repository, of Abstraction forms valuable aggregations of knowledge about the Reality.

Conception consists of Rationalisation and Realisation, both of which occur in the world of Mind, and are crucial intellectual efforts towards engineering problem-solving. Rationalisation is defined as activities to explore, understand, and represent sensible stimuli and desired intentions that explain or positively reinforce Action. It plays a significant, although not exclusive, role that binds Mind with Action and influences the way Reality is altered. Whereas Realisation is defined as activities that involve design, formation, and judgement of global plans and decisions that consist of low-level and fine-grain sequence of local activities and capabilities, which are justified, either empirically, or theoretically, in the sense that occuring behaviours in accordance with them can approximate the identified desired ends, and more importantly, improve problematic situations.

Action, involving activities in the world of Reality, consists of Implementation and Control. In contrast to thinking, reasoning, or designing activities in Mind, Implementation is about actually doing things or carrying out plans in Reality according to outputs of Realisation, and in pursuit of achieving identified desired ends from Rationalisation. Control provides run-time monitoring and management of systems during continuous interactions between systems and environments. Action is guided by Conception and influenced by Perception.

As shown in the figure, the above activities can have subordinate activities at more detailed levels. For example, Abstraction includes System Abstraction and General Abstraction; Rationalisation consists of Problem Situation and Desired End. Due to the space limitation, they are not discussed in this section, their meaning can be derived from their names and definitions of their superordinate activities, and can be understood through modelling context in section 3.2 and examples illustrated in section 4 .

It is important to note that the hierarchical structure of OAP is not rigid. Although of activities at superordinate, or more fundamental, levels are less likely to change, the nature and inner structure of activities at subordinate, or more detailed, levels can be adjusted in different context, as long as their purposes and functions still conform to those of their superordinate activities. For example, depending on whether target systems are services or embedded systems, Realisation can consist of either capability discovery and capability design, or functional design and simulation. In certain sense, superordinate activities define stable interfaces and scope for their subordinates to conform to. While the content of the latter might differs in different situations.

More importantly, OAP provides a mechanism to reuse engineering capacities, which enables and supports flexible aggregation of process activities and agile engineering in a dynamic and uncertain environment. A concept called Epitome is designed for this purpose. An Epitome of engineering capacity is a 
typical means, being generalised from proven examples, to achieve the desired result for a type of activities. It encapsulates typical engineering capacities in the form of service. For instance, based on certain Perception, a particular activity of Problem Situation recognises and represents a problematic situation. Such capacity is encapsulated in an Epitome called "Epitome 1" as depicted in figure 2. It can be used to produce the same representation of problems under the same situation, for future reference or aggregations. Such an epitome is created by generalising a proven instance of example, which is a process called Epitomisation. A collection of Epitomes provides a Horizontal Aggregation of valuable engineering assets, just like a collection of Abstraction forms valuable aggregation of knowledge about the Reality.

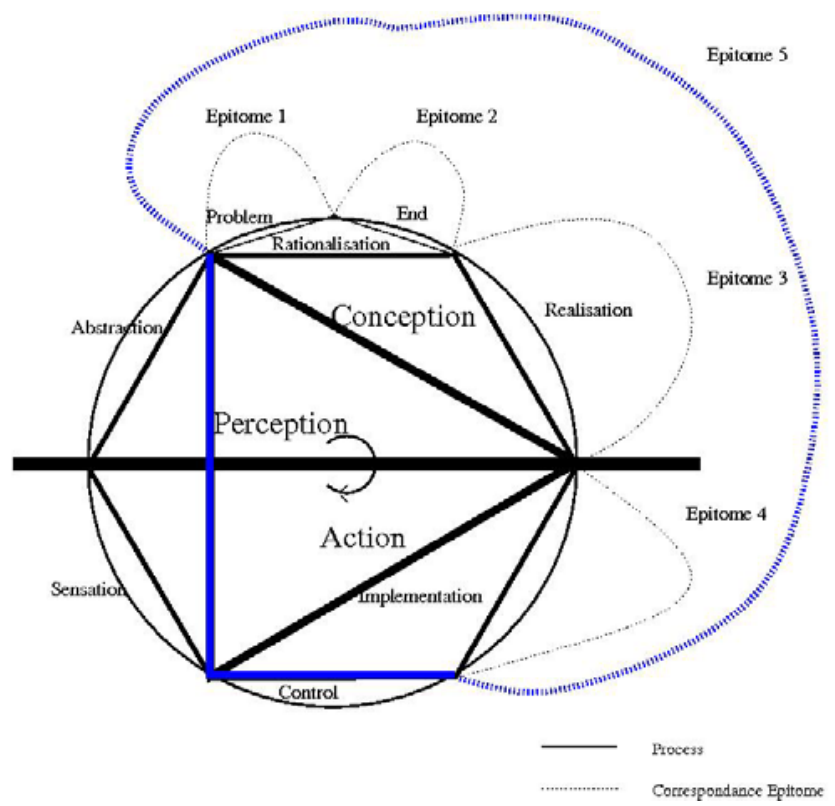

Fig. 2. An example of flexible aggregations of process using Epitomes

Moreover, Epitome can be aggregated hierarchically to form Vertical Aggregation, which can be used to create tight correspondence, or Aggregation Linkage between two process activities that would otherwise not directly relate to each other. Figure 2 depicts it. If a perceived situation has been improved through application of a series of Epitomes: from "Epitome 1" to "Epitome 4", which, respectively, encapsulates activity capacity for Problem Situation, Desired End, Realisation, and implementation at various levels, we can aggregate these Epitomes into a single one called "Epitome 5", as a typical means to generate specific ends, which creates a "strong correspondence" between a Perception of situation and desired improvements in Reality. The "strong correspondence" here means that the coherently interconnected capacities provide a typical, and proven way 
to make improvement to a particular type of problematic situations. Once justified by empirical experience, "Epitome 5" can be directly applied on a specific situation and makes desired improvement. Therefore, engineering activities can be directly aggregated together to achieve desired results without having to go through predefined subordinate or intermediate activities.

\subsection{A Model-Driven Method and Supporting Tools}

To facilitate process activities and their interactions, OAP integrates a modeldriven method in a unified fashion, in the sense that everything is supported by models within a consistent infrastructure. That is, various models, either specially-designed ones such as Problem Situation Model (PSM) and Desired End Model (DEM) in Rationalisation, or general-purpose ones such as UML Activity Diagrams, are used as specific means to explore, represent, and utilise outcomes of various activities. All OAP models such PSM and DEM are manipulated in the same way within an integrated environment.

As an example, we briefly describe the meta-model of PSM as depicted in figure 3. It consists of a number of Problems that might violate specific higherorder, or subordinate, Purposes, or be caused by some Facts that are constrained by Reality, and cause, or be caused by, other problems.

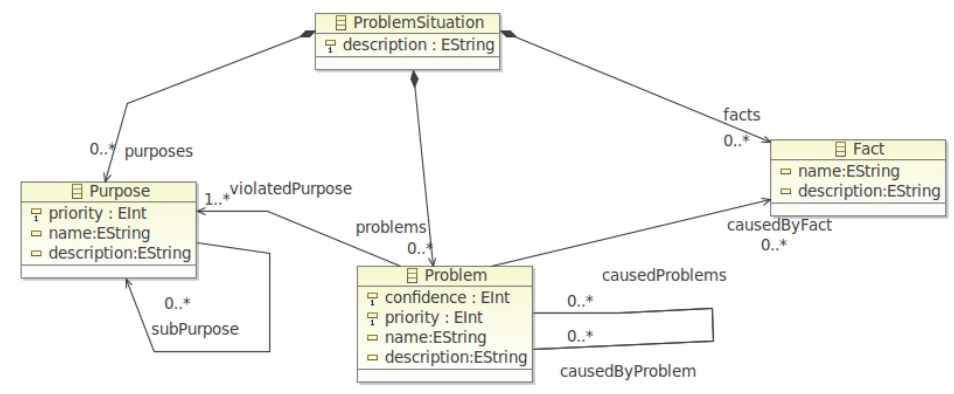

Fig. 3. The meta-model of Problem Situation

To facilitate application of OAP, a range of graphical tools have been developed, and are integrated in the Integrated Process Environment for OAP (IPEOAP). It is developed using Java programming language on top of Eclipse IDE and other Eclipse modelling Framework (EMF) supported modules. A range of graphical model editors are provided to view, create and modify models for different OAP activities, such as PSM and DEM. Example of these models and their manipulation in IPEOAP will be briefly presented in section 4. Moreover, IPEOAP also supports automatic transformation of models and systematic generation of desired artefacts. For example, PSM can be automatically transformed into documents that describe the problematic situations. Similarly, a UML activity diagram in Realisation is translated into lower-level run-time representations, such as BPEL artefacts or Java code. 


\section{A Case Study}

In order to obtain insights about the effect and value of OAP by applying its concepts, methods, and tools in a real-world setting, a controlled case study is conducted in the context of the First Home Saver Accounts (FHSA), a scheme introduced by the Australian federal government that aims to help young residents to purchase their first homes, by providing subsidy of government contributions and tax benefits. The purpose is to implement a proof-of-concept, as opposed to proof-of-performance, to demonstrate that the process meets its objectives in general, that is, a process mechanism to deal with a problematic situation in a complex and dynamic environment. A proof-of-concept system is produced using IPEOAP toolkit. Apache Tomcat web server is used to host the generated web content. Apache Axis2 Web Service Engine and ODE are used to host generated Java web services and BPEL. JBoss provides application server that contains some Java business logic. Derby Database is used for database implementation.

The OAP process started with Abstraction, which includes modelling of current business domains such as structure and process of business. UML Class Diagrams are used to model static information structure such as Customer, Bank Account, Clerk. UML Activity Diagrams are used to model business behaviour and workflow such as managing FHSA account. These models are created and managed within IPEOAP using Eclipse UML2 tools.

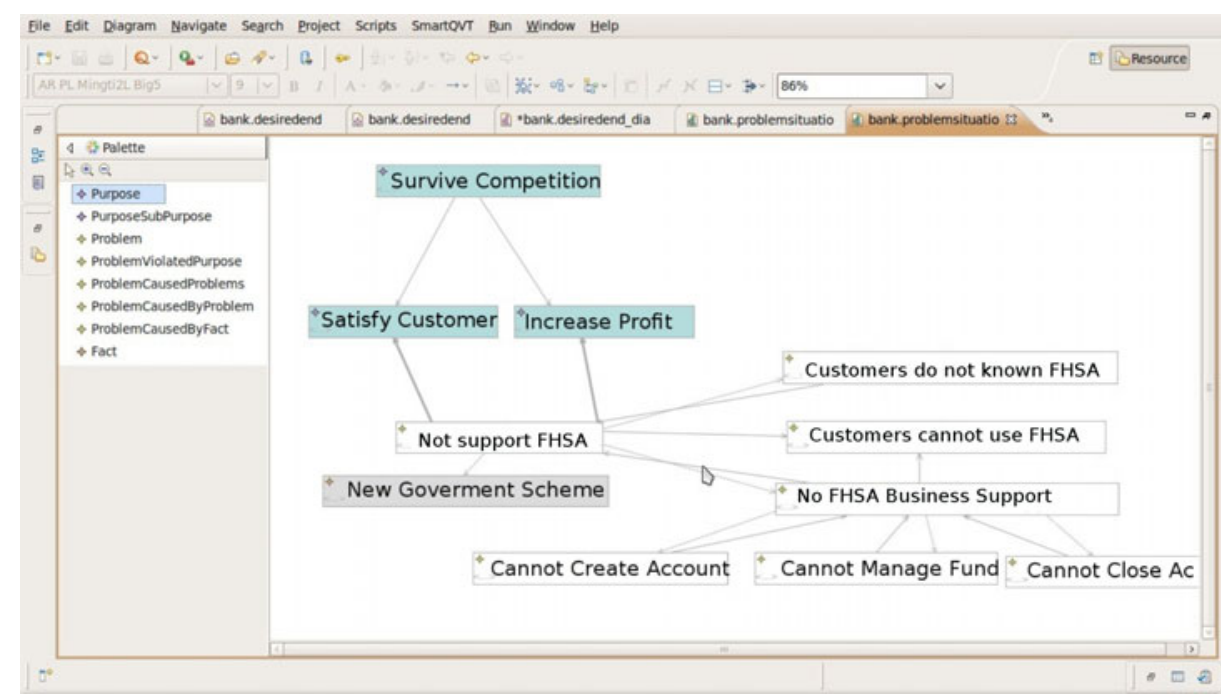

Fig. 4. An example of Problem Situation Model (PSM) in FHSA

To illustrate the concept and application of PSM in an IPEOAP environment, figure 4 shows a simple example of PSM for a financial institution that does not support FHSA scheme. It contains a higher order Purpose: namely "Survive 
competition" that has two subordinate Purposes: "Satisfy customer" and "Increase profit". It also has a high level Problem, "Not support FHSA", which violates the above Purposes. This problem is caused by a Fact: "New Goverment Scheme", that is, the government just officially released the consolidated version of policy recently and the organisation simply does not have the resources to react promptly in an uncertain market. This Problem is also caused by some other Problems. For example, many customers do not recognise the benefits of FHSA. Moreover, many of them are not eligible and the proportion of these customers remain unknown due to lack of information. Therefore, as a strategic management decision, no business support for FHSA was provided at the time of FHSA announcement. This directly leads to some subordinate Problems: "Existing customers cannot create their first FHSA account", "Cannot manage existing fund", and "Cannot terminate existing fund". This scenario exemplifies a typical complex problem situation that modern organisations are often involved in a dynamic marketing and legislation environment. As shown in the figure, IPEOAP provides a graphical editing tool that enables efficient creation and manipulation of PSM elements and their relationships. Moreover, using its transformation engine, the PSM is automatically transformed into HTML documents with informative details that describe the situation, which help business stakeholders to explore, understand, and communicate the problem situations.

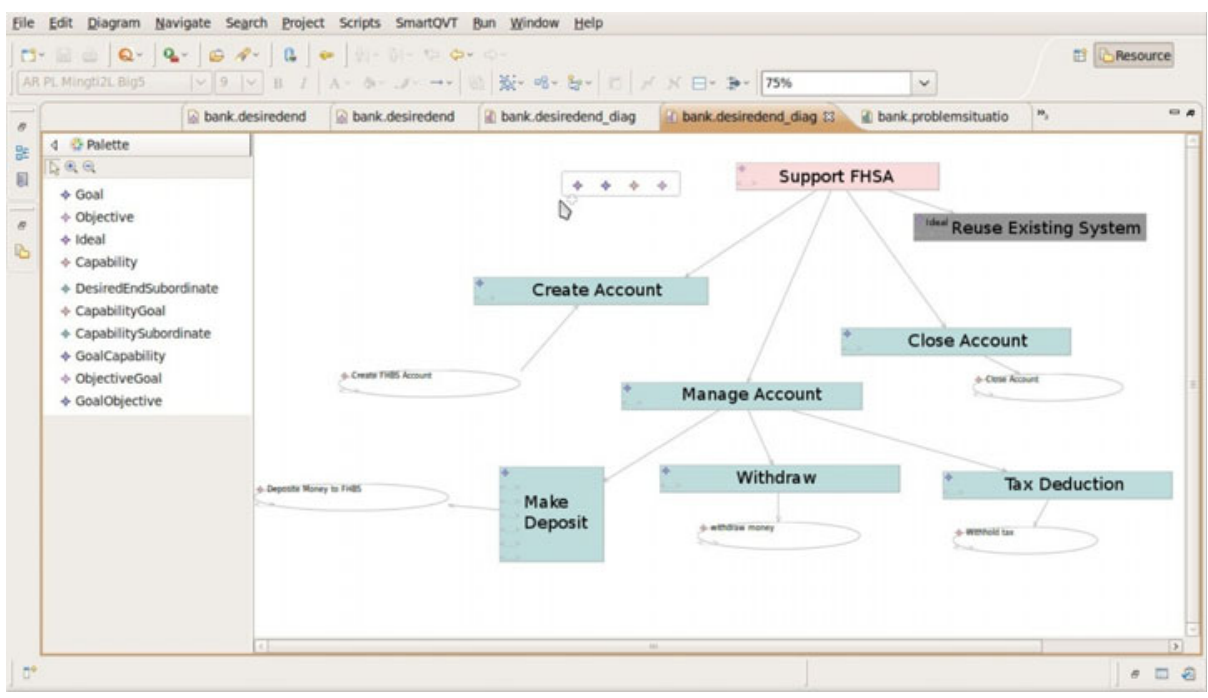

Fig. 5. An example of Desired End Model (DEM) in FHSA

The value of such a PSM lies in its function of facilitating not only discovery and communication of complex business situations and concerns at early stages of process life cycle, but also systematic derivation of relevant and sensible engineering artefacts during successive activities, such as DEM. It hence provides 
justification of and guidance for derivation of Implementation that alters the states of reality in the "right" way.

For instance, in accordance with every identified Problem in a PSM, specific DEMs are systematically derived with an aim of improving the situation. As an example, "A lack of business support for FHSA" is regarded as a critical problem in PSM from business operational point of view. A DEM is hence created as depicted by figure 5 It contains an Ideal: "Reuse existing account management system" and a more specific Objective, "Support FHSA", which has a number of concrete and operational Goals, such as "Create Account", "Close Account", and "Manage Account". The latter also contains some subordinate Goals, such as "Make Deposit", "Withdraw fund", and "Tax Deduction". Like PSM, creation and manipulation of DEM is also graphically supported by IPEOAP.

The value of DEM lies in the fact that it provides a mechanism to capture less-rigid intentions and desires that involve certain degree of uncertainty, such as ideals that are often impossible to achieve. Desired intentions are justified as long as they improve identified problematic situations at a desired level of satisfaction. Pragmatically, DEM has its value in facilitating derivation of conceptual planning and design in successive Realisation activities, which, in turn, guide, direct, or generate desired Implementation to change the reality as expected.

For example, in accordance with every Goal in above DEM, such as "Create a FHSA account", more specific Realisation models are systematically derived to satisfy these desired ends. These Realisation models, often represented as UML Activity Diagrams or BPMN models, provide detailed functional design that consists of various interconnected capabilities, such as "Check Eligibility", "Import user information from Tax office", and "Create account", which collectively satisfy the Goal of creating FHSA accounts for owners of FHSA scheme.

Finally, these abstract Realisation models, independent of specific implementation platform, are systematically, and almost automatically, translated into concrete Implementation artefacts, such as BPEL, software programs in Java programming language, or textual plan instructions, for specific implementation platform. The generated artefacts can be executed in run-time environment in Reality to satisfy the Desired End and improve identified Problem Situation. Because of advances of technologies in MDE, such as Eclipse modeling platform, transformations between abstract Realisation models such as UML Activity Diagrams and Implementation artefacts such as BPEL are relatively straightforward and are not presented in this paper.

\section{An Analysis of OAP}

The case study shows some positive results. Firstly, the modelling mechanism in OAP, such as PSM and DEM, provides an effective formalism to explore, reason about, and rationalise problematic situations, and to form rational desired ends to improve such situations. These important higher level intellectual efforts justify and foster engineering design decisions and design of plans. Exploration and clarification of problematic situation using PSM helps to identify higher 
level purposes and problems with high priority that were not initial recognised in a local problem scope. For example, a lack of FHSA business support is later confirmed as a strategic problem due to its violation with high level critical purposes. Secondly, the interrelationship between OAP activities are systematically supported by linkages between various higher level models, with aid of integrated tool environment that fosters and automates access to unified model repository and transformation engines. Thirdly, improvement to engineering productivity is observed although not formally measured against other approaches. The proofof-concept implementation enables encapsulation of knowledge and engineering capacities, and allows automatic generation of concrete documentation and systems based on a series of interconnected OAP models. Therefore, the majority of engineering efforts is designing and managing new abstract models, such as PSM and DEM as illustrated in this section. With support from IPEOAP, these modelling and transformation tasks were achieved in a short period of time.

This approach is different from Boyd's OODA loop in the sense that it is more concrete, specific, clearly defined, and generally better supported in an engineering context. For example, the boundaries, content, relationships, and value of involved activities are defined or clarified in the context of software-intensive systems engineering. Various representational formalisms, either specially designed, or general purpose, modelling language, are provided for OAP activities, with support from integrated tools. Moreover, instead of simply accelerating the pace of process, this approach provides a mechanism to reuse knowledge and capabilities in support of system flexibility and agility, which enables formation of coherent aggregation of engineering knowledge and process.

Its Rationalisation approach in Mind differs from traditional requirement engineering in terms of scope, mechanism, and "softness". Traditional requirement elicitation, analysis and management usually takes a rigid and "hard" approach, which is based on an assumption that there are requirements, clearly-definable and stable, out there to be collected in all situations. However, we believe such an assumption is not always valid in an open environment, where situations are complex, volatile, and uncertain. The common concept of "requirement" does not involve or imply "soft" intentions and desires that involve certain degree of uncertainty in problematic situations, which, undeniably, also influence the way engineering plans and design are derived and carried out. On the contrary, our approach provides a specific means to enable and support discovery, understanding and utilisation of these aspects of engineering. That is, it incorporates the concept of Desired End in the context of identified problematic situations, and systematically provides sensible targets for Realisation such as engineering planning and design. Its aim is to improve problematic situation to a satisfied degree, as opposed to achieving a "we-must-do-THIS" type of "requirements".

The proof-of-concept also reveals some issues and limitations. Currently not all parts of the OAP conceptual model are supported, such as Sensation, Test and Control. They are not incorporated appropriately in OAP at this stage because of a lack of deep understanding of their nature and characteristics. Moreover, process monitor and debugging activities are not supported, which makes it hard 
to identify problems when things go wrong. Furthermore, there are no analysis tools applied to identify syntactic issues on abstract models, which makes it less efficient to find and remove problems before model transformations get started. More importantly, no process metrics are applied to quantitatively evaluate the effectiveness of this process, especially in comparison with other approaches. Further work on these issues have been considered.

\section{Conclusion}

Aiming at addressing engineering challenges in an open world, we propose a novel engineering process called Organic Aggregation Process (OAP). It consists of: 1) a coherent and flexible conceptual model that consists of various carefully-categorised, well-organised, and inter-connected activities at various levels; 2) a model-driven method that enables and supports various activities using various specially-designed, or general-purpose, modelling and model transformation mechanisms; and 3) an integrated supporting tools environment called IPEOAP that provides unified modelling features including graphical manipulation of OAP model, and enables systematic generation of concrete systems.

The contributions of this work include: 1) It provides a coherent and flexible conceptual framework for engineering process to deal with dynamically complex open world; 2) It supports, and promotes a focus on, higher-level intellectual efforts within the world of Mind. Specifically, it provides i) a Problem Situation modelling mechanism to explore, understand, and capture complex problem situations that involve certain degree of uncertainty; and ii) a Desired End modelling mechanism to derive and represent "soft" desired ends and intentions in the context of identified problematic situations; 3) It provides a mechanism to coherently and systematically link various engineering activities, which, collectively, aim to tackle problem situations using intellectual capacities of Mind, and to form sensible actions to achieve desired improvement in Reality; 4) It provides a mechanism to capture and reuse engineering capacities and knowledge that supports flexible process aggregations and organic engineering agility.

A proof-of-concept case study is also presented in this paper to demonstrates the concepts and application of the OAP. Evidence shows that this process model can be applied in real settings and achieves its design goals in general. It helps to manage engineering processes in a complex problem situations and improve engineering efficiency in both world of Mind and Reality. However, some important limitations are also revealed, which lead to some future work, including improvement, more comprehensive empirical assessment and evaluation.

\section{References}

1. Baresi, L.: Toward open-world software: Issue and challenges. Computer 39, 36-43 (2006)

2. Aurobindo, S.: Heraclitus. Arya Publishing House, Calcutta (1941) 
3. Whitehead, A.: Process and reality: corrected edition. The Free Press, New York (1978)

4. Fuggetta, A.: Software process: a roadmap. In: Proceedings of the conference on the future of software engineering, pp. 25-34 (2000)

5. Royce, W.: Managing the development of large software systems. Proceedings of IEEE Wescon 26, 9 (1970)

6. Larman, C., Basili, V.: Iterative and incremental development: A brief history. Computer, 47-56 (2003)

7. Osinga, F.: Science, strategy and war: the strategic theory of John Boyd. Routledge Abingdon, UK (2007)

8. Beck, K., Andres, C.: Extreme programming explained: embrace change. AddisonWesley Professional, Reading (2004)

9. Shapiro, S.: Splitting the difference: The historical necessity of synthesis in software engineering. IEEE Annals of the History of Computing, 20-54 (1997)

10. Checkland, P.: Systems thinking, systems practice. J. Wiley, New York (1981) 